J. Gynäkol. Endokrinol. CH 2020 · 23:156-159 https://doi.org/10.1007/s41975-020-00168-7 Online publiziert: 4. November 2020

(c) Der/die Autor(en) 2020

\section{Daniela Chemogo-Gbellu}

Klinik für Frauenheilkunde und Geburtshilfe, Abteilung Endokrinologie und Reproduktionsmedizin, Goethe Universität, Frankfurt, Deutschland

\title{
Endometriose, aktuelle medikamentöse Therapieempfehlungen mit Schwerpunkt auf der hormonellen Therapie mit Gestagenen
}

Das Leitsymptom der Endometriose, der Unterleibsschmerz, kann zu gravierenden Einschränkungen der Lebensqualität der betroffenen Frauen führen. Der wirtschaftliche Aspekt der Endometriose durch Ausfall der Arbeitskraft und medizinische Interventionen ist beachtlich, die sozioökonomischen jährlichen Kosten pro betroffene Frau liegen in Deutschland bei ca. $9579 €$, in der Schweiz bei ca. $9630 €$, bei Frauen im Alter von 15 bis 49 Jahren [4].

Die Diagnostik der Endometriose sollte die genaue Anamnese der Beschwerden (Dysmenorrhö, prämenstruelle Schmerzen, Dyschezie, Dysurie, Hämaturie, Hämatochezie) umfassen ebenso wie die bimanuelle vaginale Untersuchung, die Spekulumeinstellung im Zweifel mit geteilten Spekula, um Herde im hinteren Scheidengewölbe nicht zu übersehen. Die vaginale Sonographie zur Erfassung von Endometriomen ist notwendig. In manchen Fällen kann eine MRT-Untersuchung bei Verdacht auf Adenomyosis uteri durchgeführt werden.

Die Sicherung der Diagnose kann einzig durch diagnostische Laparoskopie und histologische Sicherung erfolgen. Allerdings kann eine empirische Therapie bei hinreichender Symptomatik auch ohne operative Diagnostik sinnvoll sein [1].
Neben der operativen Therapie, die in der Entfernung von Läsionen des Peritoneums sowie Exstirpation von Endometriomen des Ovars und tief infiltrierender Endometrioseherde besteht (- Abb. 1), stehen verschiedene medikamentöse Therapien zur Verfügung.

Bei bestehendem Kinderwunsch ist entsprechend dem Ausprägungsgrad der Endometriose, der Belastung der Patientin und der Lokalisation der Endometrioseläsionen abzuwägen, ob dem Paar angeraten werden kann, eine Spontanschwangerschaft anzustreben. Eine gründliche Fertilitätsdiagnostik beider Partner sollte selbstverständlich sein, mit Abklärung des tubaren Faktors, dem Nachweis ovulatorischer Zyklen und der andrologischen Fertilität durch mindestens ein Spermiogramm. Therapeutisch kommen bei Dysmenorrhö oder zyklusunabhängigen Schmerzen hier Analgetika infrage.

Das Kinderwunschpaar muss gut aufgeklärt werden, insbesondere wenn das Risiko einer ovariellen Endometriose besteht oder Endometriome bereits gesichert wurden. Aufklärungsinhalt ist das erhöhte Risiko der sukzessiven Destruktion funktionellen Ovargewebes durch Endometriome mit Reduktion der ovariellen Reserve und ggf. folgenden operativen Therapien mit weiterem Verlust von Ovargewebe. Auch kann trotz gesicherter Tubendurchgängigkeit ein tu- barer Funktionsverlust die Fertilität einschränken bei ansonsten unauffälligen Parametern. Den Paaren sollten großzügig Maßnahmen der assistierten Reproduktion empfohlen werden, wenn eine Spontanschwangerschaft nicht zügig eintreten sollte.

Der der Kinderwunschpraxis zugewiesenen Patientin mit operativ gesicherter und sanierter tiefer infiltrierender Endometriose (TIE) oder Endometriomen sollte nach guter Aufklärung über die Fertilitätseinschränkung durch die fortschreitende Endometriose eine hormonelle Therapie empfohlen werden zur Rezidivprophylaxe bis zur Umsetzung einer assistierten reproduktionsmedizinischen Therapie (ART) ebenso wie in Behandlungspausen.

Sollte bei der Patientin kein aktueller, aber ein prospektiver Kinderwunsch bestehen, ist neben der Verbesserung der Lebensqualität der Patientin der Erhalt der Fertilität wichtigstes Ziel und damit eine hormonelle Therapie mit guter Verträglichkeit auch auf längere Sicht zu empfehlen. Hier können bevorzugt reine Gestagenpräparate oder kombinierte OC zur Anwendung kommen. Als Gestagenpräparat ist in Deutschland Dienogest $2 \mathrm{mg}$ für diese Indikation zugelassen. Im „offlabel use "kann jedoch jedes Gestagen gewählt werden, allein oder in Kombination als OC, welches ggf. erwünschte Partialwirkungen aufweist (z. B. antian- 
drogene Wirkung zur Therapie bei Seborrhö und/oder Hirsutismus).

Bei abgeschlossener Familienplanung steht die gesamte Bandbreite der medikamentösen und operativen Therapien zur Wahl, hier sollte Berücksichtigung finden: der Wunsch nach sicherer Verhütung, die Verträglichkeit der Medikation, die Ausprägung der Endometriose sowie das individuelle Risiko der Patientin (Übergewicht, Nikotinkonsum, Alter, Thrombophilie).

Die bewährten medikamentösen Therapien der Endometriose umfassen hormonelle und analgetische Therapeutika.

Zur hormonellen Therapie geeignet sind nach Empfehlung der geltenden Leitlinie der European Society of $\mathrm{Hu}$ man Reproduction and Embryology (ESHRE) aus dem Jahr 2013 (zuletzt bearbeitet vor fünf Jahren, aktuell unter Überarbeitung)

- hormonelle Kontrazeptiva (zyklisch oder im Langzyklus),

- Gestagene (oral oder in Form eines intrauterinen Pessars),

- Antigestagene und Gonadotropin-Releasing-Hormon-Agonisten (GnRH-Agonisten) und

- Aromataseinhibitoren [1].

Gestagene wirken durch eine direkte Hemmung der Endometriumproliferation, transformatorisch und antiinflammatorisch.

GnRH-Analoga, welche lange als Standardtherapie bei Endometriosepatientinnen eingesetzt wurden, sind zwar sehr effektiv in ihrer Wirksamkeit, supprimieren jedoch die Östradiolspiegel auf postmenopausales Niveau mit Symptomen eines Östrogenmangels. Vasomotorische Beschwerden, Trockenheit der Scheide mit Dyspareunie, Libidoverlust und Verlust von Knochensubstanz mit dem Risiko der Osteoporose erfordern die ergänzende „Add-backTherapie“ (niedrig dosierte Östrogengabe) insbesondere bei Anwendungen über 6 Monate.

Die Therapie mit Analgetika ist rein symptomatisch ohne einen Effekt auf die Endometriose und deren Proliferation. Der Einsatz ist gerechtfertigt, wenn andere Behandlungsoptionen nicht ge-

J. Gynäkol. Endokrinol. CH 2020 · 23:156-159 https://doi.org/10.1007/s41975-020-00168-7

(c) Der/die Autor(en) 2020

\section{Chemogo-Gbellu}

\section{Endometriose, aktuelle medikamentöse Therapieempfehlungen} mit Schwerpunkt auf der hormonellen Therapie mit Gestagenen

\section{Zusammenfassung}

Unter Endometriose versteht man das Auftreten von endometrialen Zellen und Zellverbänden außerhalb des Cavum uteri, welche dem hormonellen Zyklus unterliegen und zu rezidivierenden Beschwerden führen können. Die Inzidenz wird je nach Quelle mit 2-15\% der Frauen im reproduktionsfähigen Alter angegeben. Schmerzen und
Fertilitätseinschränkung sind die führenden Symptome. Unter Kinderwunschpatientinnen liegt die Inzidenz bei $20-48 \%$.

\section{Schlüsselwörter}

Schmerz · Kinderwunsch - Ovarielle Reserve . Endometriome $\cdot$ Rezidiv $\cdot$ Synthetische Gestagene

\section{Endométriose, recommandations actuelles concernant la pharmacothérapie et plus particulièrement le traitement hormonal progestatif}

\section{Résumé}

L'endométriose est l'apparition de cellules endométriales et de tissu endométrial hors de la cavité utérine, qui sont soumises au cycle hormonal et peuvent causer des symptômes récidivants. Selon les sources consultées, l'incidence est évaluée à 2-15\% des femmes en âge de procréer. La douleur et une fertilité restreinte sont les principaux symptômes.
L'incidence chez les patientes dans le domaine de la procréation médicalement assistée se situe entre 20 et $48 \%$.

\section{Mots clés}

Douleur · Désir d'enfant · Réserve ovarienne Endométriomes · Récidive · Progestatifs synthétiques wünscht, nicht verträglich oder nicht effektiv sind.

Aktuell ist die alleinige Gabe von Gestagenen noch vor der Gabe eines kombinierten oralen Kontrazeptivums im „off label use “ bei entsprechender Verträglichkeit die hormonelle Therapie der ersten Wahl.

Neben der o. g. Wirkung von Gestagenen auf Wachstum und Proliferation endometrialer Zellen wird ein weiterer Effekt synthetischer Gestagene diskutiert:

Es scheint, dass eine Resistenz von Endometriosezellen auf das natürliche Progesteron bedeutsam für die Entstehung der Endometriose ist, wobei der genaue Mechanismus weiterer Klärung bedarf: Studien konnten zeigen, dass in Endometrioseläsionen der physiologische Effekt von Progesteron auf Östrogen, der über Enzyminduktion die Umwandlung von Östradiol in Östron bewirkt, ausbleibt [2].

Östradiol als biologisch stark wirkendes Östrogen wird nicht in das deutlich weniger wirksame Östron umgewandelt [2]. Das Hormonungleichgewicht aus Östrogendominanz und niedrigen Progesteronwerten in den Endometrioseläsionen begünstig in der Folge Entzündungen und Schmerzen.

Auch die Dichte an Progesteronrezeptoren, die die Wirkung am Endometrium vermitteln und im natürlichen Zyklus typischen Schwankungen unterliegen, ist in endometriotischem Gewebe verändert. Der Progesteronrezeptor (PR-B), der die Progesteronwirkung auf das Endometrium vermittelt, ist völlig abwesend, die Anzahl der Isoform des Progesteronrezeptors (PR-A), der antagonisierend auf Rezeptor PR-B wirkt, ist erniedrigt [3].

Synthetische Gestagene scheinen eine günstigere Affinität zu den Progesteronrezeptoren zu haben und können dadurch die antiöstrogene Wirkung über die oben beschriebene Enzyminduktion ausüben. Synthetische Gestagene, die theoretisch zur Verfügung stehen, sind Chlormadinon, Cyproteron, Dienogest, 


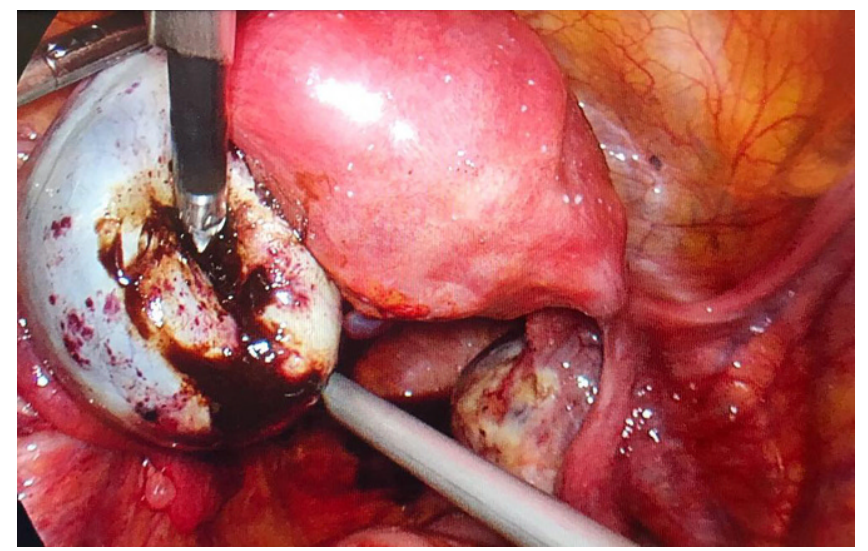

Abb. $1<$ Endometriom des Ovars. Nach Eröffnung entleert sich der typische bräunliche Inhalt, der im Deutschen Sprachgebrauch zu dem Begriff "Schokoladenzyste" führte. Zur Verfügung gestellt von Dr. M. Tamasbi Rad, Frauenklinik der Goethe-Universität Frankfurt

Desogestrel, Drospirenon, Dydrogesteron, Etonogestrel, Gestoden, Levonorgestrel, Medrogeston, Medroxyprogesteron, Megestrol, Nomegestrol, Norethisteron, Norgestimat, Norelgestromin, Tibolon und Trimegeston.

Welche der synthetischen Gestagene sollten nun bevorzugt zur Anwendung kommen? Dies hängt unter Umständen auch von der Art und Lokalisation der Endometriose ab:

Östrogen- und Progesteronrezeptoren der rektovaginalen Endometriose unterscheiden sich deutlich von jenen der peritonealen Endometrioseherde [5].

In einer aktuellen Studie wurde die Effizienz eines etonogestrelhaltigen Implantats bei symptomatischen Patientinnen mit rektovaginaler Endometriose untersucht [6]. Eingeschlossen in die o.g. Studie wurden 43 Frauen mit sonographisch nachgewiesener rektovaginaler Endometriose. Nach jeweils 6, 12 und 24 Monaten wurden mithilfe der visuellen analogen Schmerzskala sowie dem Endometriosis Health Profile (EHP-30) und einer vergleichenden computergesteuerten Bildgebung die subjektive Schmerzbelastung, die allgemeine Lebensqualität und die Ausdehnung der Endometrioseherde evaluiert.

Unter der Behandlung zeigte sich eine rasche Reduktion der chronischen Schmerzintensität („non-menstrual pelvic pain“), der Dysmenorrhö, der Dyspareunie und der Dyschezie.

Nach 6 Monaten zeigte sich in allen erfassten Domänen der EHP-30 Scale in Bezug auf die Lebensqualität im Vergleich zu den Ausgangswerten eine Verbesserung. Im Folgenden profitierten lediglich
Patientinnen mit hohem Schmerzscore (12 und mehr auf der visuellen Schmerzskala) von weiteren Verbesserungen hinsichtlich der Schmerzempfindung.

Das Volumen der rektovaginalen Endometrioseherde war nach 6 Monaten signifikant verringert, eine weitere Volumenreduktion konnte nach 12 und 24 Monaten beobachtet werden. Dies alles bei guter Verträglichkeit des Implantats [6].

Mit der Fragestellung, ob Dienogest die beste Medikation in der Therapie ovarieller Endometriose (Endometriome) sei, wurden die Ergebnisse einer Casecontrol-Multicenterstudie vorgestellt.

81 Patientinnen mit ovarieller Endometriose (Endometriomen) erhielten entweder Dienogest $2 \mathrm{mg}$ (DNG) tgl. oder zyklisch ein orales kombiniertes Hormonpräparat mit $2 \mathrm{mg}$ Dienogest und $30 \mu \mathrm{g}$ Ethinylöstradiol (EE + DNG).

Ziel der Studie war die Erfassung des Erfolgs bei der Größenreduktion der Endometriome. Ebenfalls erfragt wurden bei symptomatischen Patientinnen die Auswirkungen der Medikationen auf das Schmerzempfinden mittels der visuellen analogen Schmerzskala.

In beiden Gruppen zeigte sich eine signifikante Reduktion der Schmerzempfindung ohne statistisch relevante Unterschiede.

Hinsichtlich des Volumens der Endometriome zeigte sich jedoch nur in der DNG-Gruppe eine signifikante Größenreduktion [7].

Die Monotherapie mit DNG $2 \mathrm{mg}$ täglich sollte hinsichtlich des Erhalts funktionellen Ovargewebes und damit auch des Erhalts der Fertilität gerade der Frau mit prospektivem Kinderwunsch empfohlen werden. Eine gute Aufklärung über die Wirkweise und den günstigen Effekt auf die Fertilität kann die Compliance erhöhen und Abbrüchen aufgrund von Nebenwirkungen wie vaginalen Blutungen (Spotting und Durchbruchblutung), Kopfschmerzen, Gewichtszunahme, Depressionen, Libidoverlust, Akne, Alopezie, Schlafstörungen und vaginaler Trockenheit [8] entgegenwirken.

Im Rahmen der diesjährigen ESHRE 2020 wurde die folgende Studie mit der Fragestellung „Reduziert die postoperative Gabe von Norethisteronacetat (NETA) das Rezidivrisiko der Endometriose?" vorgestellt. Eingeschlossen in die Studie, die 2014 initiiert wurde und über 5 Jahre lief, waren 437 Frauen, bei denen eine TIE zuvor operativ therapiert worden war. Die Studienteilnehmerinnen erhielten entweder eine hormonelle Dauertherapie mit Norethisteronacetat (NETA), unmittelbar postoperativ begonnen, oder keine Medikation. In halbjährlichen Intervallen erfolgten eine vaginalsonographische Untersuchung und eine Erfassung der Schmerzsymptomatik. Im Ergebnis der Studie zeigte sich, dass die dauerhafte Gabe von NETA im Vergleich zu keiner hormonellen Therapie sowohl das Rezidivrisiko als auch die endometrioseassoziierten Symptome signifikant reduziert [9].

\section{Fazit für die Praxis}

Nach Sicherung der Diagnose ist die Therapieempfehlung unter Berücksichtigung der Lebenssituation sowie der Akzeptanz der Patientin zu wählen. Mit synthetischen Gestagenen besteht die Möglichkeit, nahezu jeder betroffenen Patientin je nach aktuellem Bedarf angepasst an ihre Lebenssituation eine gut wirksame medikamentöse Therapie anzubieten mit im Vergleich zu GnRH-Analoga geringerem Nebenwirkungsprofil. Mit der Wahl des jeweiligen Gestagens kann zudem ein günstiger Effekt auf die Größe der Läsion und das Rezidivrisiko nach operativer Sanierung je nach Ausbreitung und Lokalisation der Endometriose erreicht werden. 


\section{Korrespondenzadresse}

Daniela Chemogo-Gbellu
Klinik für Frauenheilkunde
und Geburtshilfe, Abteilung
Endokrinologie und
Reproduktionsmedizin,
Goethe Universität
Theodor-Stern-Kai 7,
60590 Frankfurt, Deutschland
Daniela.Chemogo-Gbellu@
kgu.de

Funding. Open Access funding enabled and organized by Projekt DEAL.

\section{Einhaltung ethischer Richtlinien}

Interessenkonflikt. D. Chemogo-Gbellu gibt an, dass kein Interessenkonflikt besteht.

Für diesen Beitrag wurden von der Autorin keine Studien an Menschen oder Tieren durchgeführt. Für die aufgeführten Studien gelten die jeweils dort angegebenen ethischen Richtlinien.

Open Access. Dieser Artikel wird unter der Creative Commons Namensnennung 4.0 International Lizenz veröffentlicht, welche die Nutzung, Vervielfältigung, Bearbeitung, Verbreitung und Wiedergabe in jeglichem Medium und Format erlaubt, sofern Sie den/die ursprünglichen Autor(en) und die Quelle ordnungsgemäß nennen, einen Link zur Creative Commons Lizenz beifügen und angeben, ob Änderungen vorgenommen wurden.

Die in diesem Artikel enthaltenen Bilder und sonstiges Drittmaterial unterliegen ebenfalls der genannten Creative Commons Lizenz, sofern sich aus der Abbildungslegende nichts anderes ergibt. Sofern das betreffende Material nicht unter der genannten Creative Commons Lizenz steht und die betreffende Handlung nicht nach gesetzlichen Vorschriften erlaubt ist, ist für die oben aufgeführten Weiterverwendungen des $\mathrm{Ma}$ terials die Einwilligung des jeweiligen Rechteinhabers einzuholen.

Weitere Details zur Lizenz entnehmen Sie bitte der Lizenzinformation auf http://creativecommons.org/ licenses/by/4.0/deed.de.

\section{Literatur}

1. Dunselman GA, Vermeulen $N$, Becker $C$, Calhaz Jorge C, D'Hooghe T, De Bie B, Heikinheimo O, Horne AW, Kiesel L, Nap A, Prentice A, Saridogan E, Soriano D, Nelen W (2014) ESHRE guideline: management of women with endometriosis. Hum Reprod 29(3):400-412. https://doi.org/10.1093/ humrep/det457

2. Bulun SE (2009) Endometriosis. N Engl J Med 360(3):268-279. https://doi.org/10.1056/ NEJMra0804690

3. Marquardt RM, Kim TH, Shin JH, Jeong JW (2019) Progesterone and estrogen signaling in the endometrium: What goes wrong in Endometriosis?
Int J Mol Sci 20(15):3822. https://doi.org/10.3390/ ijms20153822

4. Simoens S, Hummelshoj L, Dunselman G, BrandesI, Dirksen C, D'Hooghe T, EndoCost Consortium (2011) Endometriosis cost assessment (the EndoCost study): a cost-of-illness study protocol. Gynecol Obstet Invest 71(3):170-176. https://doi. org/10.1159/000316055

5. Götze F (2014) Endometriose und Beckenschmerzen.St. Galler Beckenbodensymposium.

6. Ferrero S, Scala C, Ciccarelli S, Vellone VG, Barra F (2020) Treatment of rectovaginal endometriosis with the etonogestrel-releasing contraceptive implant. Gynecol Endocrinol 36(6):540-544. https://doi.org/10.1080/09513590.2019.1689552

7. AngioniS, Pontis A, MaluneME, Cela V, LuisiS, LittaP, Vignali M, Nappi $L$ (2020) Is dienogest the best medical treatment for ovarian endometriomas? Results of a multicentric case control study. Gynecol Endocrinol 36(1):84-86. https://doi.org/ 10.1080/09513590.2019.1640674

8. Ebert AD, Vitezica I, von Kleinsorgen C, Wilson $T$, Halis G, Mechsner S, Magalov IS, Younis M, Makarenko TA (2011) Dienogest (DNG) in der Therapie der Endometriose. J Gynäkol Endokrinol 21(2):7-12

9. Ferrero S, Scala C, Evangelisti G, Barra F (2020) Long term treatment with noretindrone acetate decreases the postoperative recurrence of deep endometriosis at long-term follow-up. ESHRE. (Session 05: Endometriosis and uterine disorders. New clinical insights. Selected oral communications. Presentation number:0-022)

Hinweis des Verlags. Der Verlag bleibt in Hinblick auf geografische Zuordnungen und Gebietsbezeichnungen in veröffentlichten Karten und Institutsadressen neutral.

\section{Schmerz - eine Herausforde- rung}

Ein Ratgeber für Betroffene und Angehörige

3., vollst. akt. u. erw. Aufl. 2020, XVII, 204S. 52 Abb.

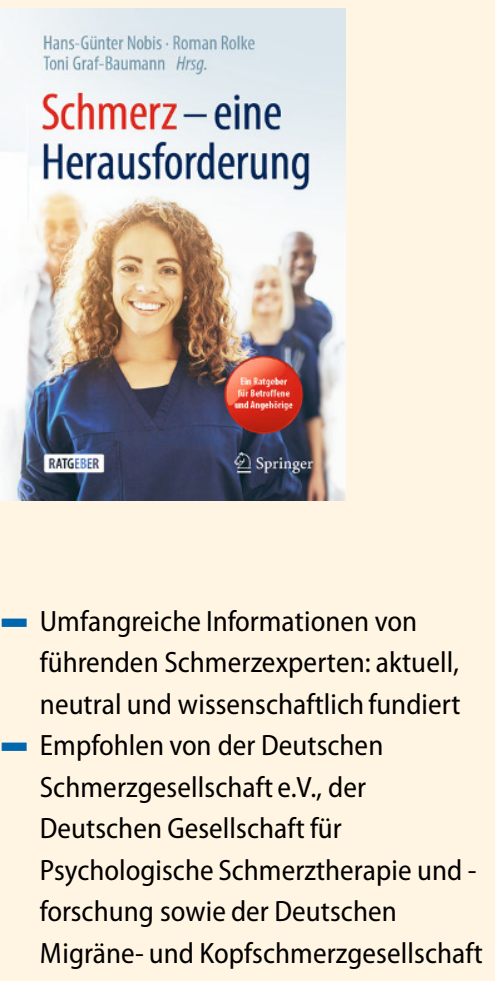

Bei mehr als der Hälfte aller Menschen mit chronischem Schmerz dauert es mehr als 2 Jahre, bis sie eine wirksame Schmerzbehandlung erhalten. Dieses Buch hilft Betroffenen, sich gezielter professionelle Unterstützung zu suchen. Knapp 60 führende Schmerzexperten haben mit über 77 Beiträgen daran mitgewirkt. Betroffene und Angehörige erfahren mehr über die körperlichen, psychischen und sozialen Zusammenhänge von Schmerz, um die Behandlung motiviert und eigenverantwortlich mitzugestalten. So versteht der Betroffene die bio-psycho-sozialen Zusammenhänge von Schmerz aus Sicht der aktuellen Schmerzmedizin und -psychologie. Die 3. Auflage wurde komplett aktualisiert und umfassend erweitert, u.a um die Themen "Schmerz und Sexualität", "Wachstumsschmerzen bei Kindern", "Endometriose" und „Gelenkschmerz", ,Naturheilkunde bei Schmerz" und was bei der Einnahme von Schmerzmitteln während der Schwangerschaft, Stillzeit, auf Reisen und beim Sport zu beachten ist. 